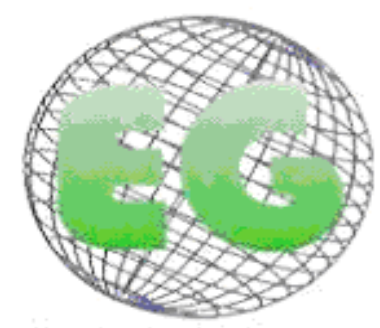

ISSN 1695-6141 $\mathrm{N}^{\circ} 27$
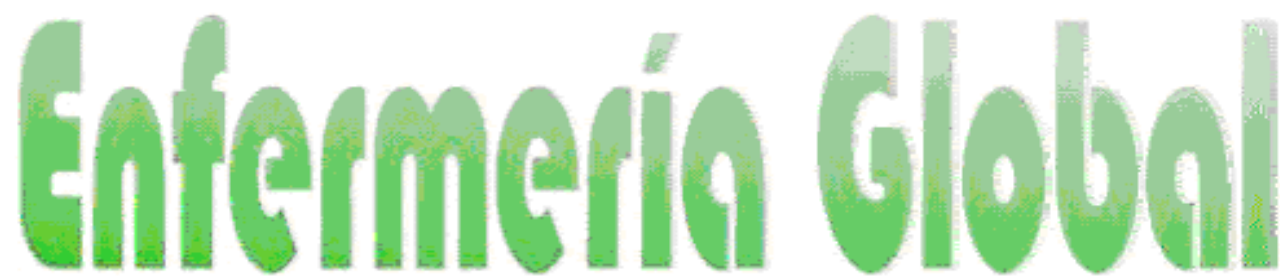

Revista electrónica trimestral de Enfermería

Julio 2012

www.um.eslegloball

\title{
Reacciones adversas: indicadores de resultados según la percepción de las enfermeras de un hospital centinela
}

Eventos adversos: indicadores de resultados segundo a percepção de enfermeiros de um hospital centinela

\section{${ }^{*}$ Queiroz Bezerra, AL., ${ }^{* *}$ Queiroz, E dos S., ${ }^{* * *}$ Weber, J., ${ }^{* * * *}$ Tanferri de Brito Paranaguá, T.}

\begin{abstract}
*Doctora en Enfermería. Profesora Asociada de la Facultad de Enfermería de la Universidad Federal de Goiás. E-mail: analuciaqueiroz@uol.com.br $\quad$ **Enfermera. Residente en Enfermería Oncológica del Hospital Araújo Jorge. Goiânia, Goiás. *** Enfermera asistencial del Hospital Unique. Goiânia, Goiás. ****Enfermera. Mestranda del Programa de Pós-Graduación en Enfermería de la Facultad de Enfermería de la Universidad Federal de Goiás. Brasil.
\end{abstract}

Palabras clave: enfermería; administración de riesgos; efectos adversos; iatrogenia.

Palavras-chave: enfermagem; gerenciamento de riscos; eventos adversos; iatrogenia.

Keywords: nursing; safety management; adverse events; iatrogenic disease.

\section{RESUMEN}

El presente estudio tiene como objetivo determinar la comprensión de las enfermeras que trabajan en un hospital centinela de eventos adversos, identificar los eventos más frecuentes y las medidas adoptadas por la institución para la seguridad de la atención, desde el punto de vista de las enfermeras. Estudio descriptivo, exploratorio, realizado durante el año 2009 en un hospital universitario en el Medio Oeste con 50 enfermeras que respondieron a un cuestionario. Los datos se analizaron estadísticamente, con las frecuencias absolutas y relativas. Los eventos adversos más frecuentes fueron las infecciones hospitalarias, error de medicación, trauma durante procedimientos, úlceras por presión, caídas y quemaduras. La mayoría comprende el concepto de eventos adversos, aunque algunos han señalado las dificultades. Todos reconocieron los riesgos y señalaron la adopción de medidas de acompañamiento y evaluación del servicio para mejorar la práctica asistencial.

\section{RESUMO}

O presente estudo tem como objetivo verificar o entendimento dos enfermeiros atuantes em um hospital sentinela sobre os eventos adversos, identificar os eventos mais frequentes e as medidas adotadas pela instituição para a segurança da assistência, segundo a ótica destes enfermeiros. Estudo descritivo, exploratório realizado durante o ano de $2009 \mathrm{em}$ um hospital de ensino do Centro Oeste com 50 enfermeiros que responderam um questionário estruturado. Os dados foram analisados estatisticamente, apresentando as freqüências absolutas e relativas. Os eventos adversos mais citados foram infecção hospitalar, erro de medicação, trauma durante procedimentos, úlcera de pressão, queda e queimadura. A maioria entende o 
conceito de eventos adversos, entretanto alguns apontaram dificuldades. Todos demonstraram reconhecer os riscos e apontaram a adoção de medidas de acompanhamento e avaliação do serviço para a melhoria da prática em saúde.

\section{ABSTRACT}

This study aims to determine the understanding of nurses working at a sentry hospital of adverse events, to identify the most frequent events and the actions taken by the institution for the safety of care, from the viewpoint of nurses. This is a descriptive, exploratory study conducted during 2009 in a teaching hospital in the Midwest with 50 nurses who answered to a questionnaire. The data were analyzed statistically, featuring the absolute and relative frequencies. Adverse events most frequently cited were hospital infection, medication error, trauma during the procedure, pressure ulcers, falls and burns. Most of them understand the concept of adverse events, though some have pointed out the difficulties. All of them recognized the risks and noted the adoption of measures for monitoring and evaluating the service to improve health care practice.

\section{INTRODUCCIÓN}

El acto de cuidar tiene como objetivo reducir o eliminar el sufrimiento del paciente aunque también puede causar eventos adversos. Eventos adversos son incidentes que ocurren durante la prestación del cuidado de la salud, no relacionados con la enfermedad básica, y que acaba en daños al paciente, pudiendo generar una afectación de la estructura o función corporal y/o algún efecto nocivo, como enfermedad, lesión, incapacidad, o muerte, pudiendo este ser físico, social y psicológico ${ }^{(1)}$.

Estudio de la Práctica Médica de Havard (HMPS) desarrollado en California en 1974 con más de 20.000 ingresos hospitalarios, verificó la ocurrencia de eventos adversos en 4,65\% de los ingresos. Ya en 1992, un análisis de la Calidad en la Atención Médico-hospitalaria Australiana, que revisó 14.179 registros de pacientes seleccionados aleatoriamente de 31 hospitales, indicó que en $16,6 \%$ de los ingresos ocurrió por lo menos un evento adverso; $13,7 \%$ de los eventos acabaron en incapacidad permanente; y 4,9\% se asociaron a muerte ${ }^{(2,}$ 3).

Datos de la Organización Mundial de la Salud indican que de cada 10 personas que precisan de cuidado en salud, por lo menos una sufrirá agravamiento derivado de eventos $\operatorname{adversos}^{(1)}$.

En Brasil, el análisis de tres hospitales docentes de Rio de Janeiro permitió identificar que, aproximadamente, $8 \%$ de los pacientes internados sufrieron uno o más eventos adversos, siendo que alrededor del $67 \%$ de estos eventos podían haberse evitado. Este estudio también mostró una diferencia significativa en la incidencia de eventos adversos evitables en Brasil cuando comparado con países desarrollados ${ }^{(4)}$.

Estudio realizado en un hospital centinela, en el sector de gerencia de riesgos, analizó 100 fichas de notificaciones, el total notificado durante tres años, evidenciando denuncias, fallos en la cumplimentación de los formularios y ausencia de informaciones sobre el evento, verificando el desconocimiento de los profesionales sobre la importancia de notificar y la necesidad de estimular la notificación completa de los eventos adversos y el desarrollo de una cultura de seguridad ${ }^{(5)}$.

Como señalan los datos, son muchas las personas víctimas de eventos adversos, representando un grave perjuicio financiero. En Gran Bretaña, el costo de eventos adversos evitables es de aproximadamente US\$1500 millones por año, solamente por la ocupación adicional de cama hospitalaria. Costos más amplios de tiempo de trabajo perdido, 
indemnización por incapacidad, entre otros, son aún mayores ${ }^{(6,7)}$. En los EUA, los costos anuales provocados por estos eventos varían de 1700 a 2900 millones de dólares ${ }^{(8)}$.

La publicación del libro To err is human: building a safer health care system, del Institute of Medicine, en 2000, y los informes del Committee on Quality of Health Care in America, de los EUA, con datos sobre la mortalidad relacionada con errores derivados del cuidado de salud que podrían ser evitados, culminó en interés mundial por la seguridad del paciente, siendo considerado también un problema de salud pública en varios países desarrollados ${ }^{(6,}$ 9).

Se define seguridad en la asistencia de la salud como ejecución de una práctica libre de daños, realizada por profesionales capacitados y que fundamenten sus acciones en la busca continua de calidad, en ambiente asistencial permeado por cultura organizacional no punitiva, receptiva y flexible y que posibilite cambios para la prevención de los errores ${ }^{(10)}$.

Si consideramos al enfermero como responsable de la coordinación y administración de todo el proceso de asistencia desarrollado con el paciente, su asistencia debe ser realizada eficientemente, garantizando la calidad del cuidado prestado y, principalmente, la satisfacción del paciente y sus familiares ${ }^{(11)}$.

De esta forma, el alma de la práctica enfermera está en atender las necesidades del paciente y de su família, teniendo como praxis el cuidado integral e individual del ser humano. Tales presupuestos deben fundamentar prácticas innovadoras, basadas en evidencias científicas, destacando la necesidad de integración de las actividades de todos los miembros del equipo de enfermería, aplicando los conocimientos provenientes de investigaciones científicas actualizadas y de calidad por profesionales competentes, respetando los valores y deseos de pacientes y familiares ${ }^{(9)}$.

Así, conocer y entender sobre los eventos adversos, su gerenciamiento, control y factores de riesgo, permite al enfermero y a todo el equipo multidisciplinar, la implementación de medidas preventivas y tratamientos eficaces.

En este sentido, la investigación sobre los eventos adversos lleva al conocimiento de fallos latentes del proceso de cuidar, con el objetivo de contribuir a la implementación de estrategias que reduzcan y eviten los fallos identificados, minimizando su impacto y contribuyendo a realizar una práctica segura ${ }^{(6,10,12-14)}$

Considerando la enfermería como la mayor fuerza de trabajo dentro de las instituciones de salud brasileñas y del mundo y dada su importancia en la prevención de los eventos adversos, de su responsabilidad para garantizar la seguridad del paciente y la calidad de los servicios, destaca la relevancia y necesidad de investigar el conocimiento de estos profesionales sobre la ocurrencia de los eventos adversos dentro de la realidad en que se insertan, así como las medidas de seguridad practicadas por estos profesionales, con la intención de propiciar las acciones desarrolladas por la enfermería, garantizando una asistencia de calidad con el mínimo de riesgos.

Ante lo expuesto, el presente estudio tiene por objetivo verificar la comprensión de los enfermeros actuantes en un hospital centinela sobre los eventos adversos, identificar los eventos adversos más frecuentes y las medidas de seguridad adoptadas por la institución para la seguridad de la asistencia, según la óptica de estos enfermeros. 


\section{METODOLOGÍA}

Estudio descriptivo, exploratorio, realizado en un hospital de enseñanza del Centro Oeste realizado entre octubre y diciembre de 2009. La institución se caracteriza por la prestación de atención de alta complejidad, desarrollo de actividades de enseñanza, investigación y extensión y por ser integrante del proyecto Hospitales Centinela, tiene por finalidad la notificación de eventos adversos y quejas técnicas de productos en uso en los servicios de salud.

El Proyecto Hospitales Centinela se creó por la Agencia Nacional de Vigilancia Sanitaria en 2001, actuando en el área de seguridad del paciente, con el objetivo de medir y estimular la notificación de eventos adversos y quejas técnicas de productos en uso en los servicios de salud. Así, una de los fines a cumplir por los prestadores de cuidados para medir y evaluar la calidad de la asistencia prestada al paciente es la ocurrencia de eventos adversos ${ }^{(15-16)}$.

La población está compuesta por 50 enfermeros que actúan en las diversas unidades de la institución, que aceptaron participar en el estudio. Los criterios de selección fueron acessibilidad, aceptación y estar trabajando en el período de colecta de datos.

La colecta se realizó en el propio lugar de trabajo de los enfermeros utilizando un cuestionario estructurado con preguntas objetivas y subjetivas, relacionadas con la caracterización de los sujetos, la comprensión de eventos adversos, los eventos adversos más frecuentes en la unidad donde el enfermero actúa y medidas de prevención de eventos adversos abordadas en el programa de educación continuada.

Se realizó análisis estadístico y descriptivo de los datos, presentados en forma de gráficos y tablas con frecuencias simples y relativas, discutidos con literatura científica específica.

Se informó a los participantes sobre los objetivos del estudio y tras la garantía de anonimato y sigilo de las informaciones firmaron el Término de Consentimiento Libre y Aclarado. Para los demás aspectos éticos siguieron las recomendaciones del Consejo Nacional de Salud, Resolución 196/96 ${ }^{(17)}$. Proyecto aprobado por el Comité de Ética en Pesquisa del campo en estudio (Protocolo № 100/2007).

\section{RESULTADOS Y DISCUSIÓN}

Participaron en este estudio 50 enfermeros, caracterizados conforme se muestra en la Tabla I.

Tabla I- Perfil profesional de los enfermeros de un hospital centinela. Goiânia, Goiás, Brasil, 2010.

\begin{tabular}{lcc}
\hline Variable & $\mathrm{n}$ & $\mathrm{F}$ \\
\hline Sexo & & \\
Femenino & 43 & $86 \%$ \\
Masculino & 7 & $14 \%$ \\
& & \\
Edad & & \\
$20-25$ años & 3 & $6 \%$ \\
$26-30$ años & 5 & $10 \%$ \\
$31-35$ años & 5 & $10 \%$ \\
$36-40$ años & 15 & $30 \%$
\end{tabular}


41-45 años

46 o más años

\section{Tiempo de formado}

1-5 años

6-10 años

11-15 años

16-20 años

20 o más años

\section{Titulación}

Bachillerato

Especialista

Máster

\section{Tiempo de servicio en la} institución

1-5 años

6-10 años

11-15 años

16-20 años

20 o más años

\section{Turno de trabajo}

Matutino

Vespertino

Nocturno

Matutino y vespertino

Matutino y nocturno

Todos los turnos

\section{Contrato de trabajo}

Uno

Dos

Más de dos
$1224 \%$

$1020 \%$

$1326 \%$

$1020 \%$

$918 \%$

$1326 \%$

$5 \quad 10 \%$

$10 \quad 20 \%$

$33 \quad 66 \%$

$7 \quad 14 \%$

\section{Total}

$1938 \%$

$5 \quad 10 \%$

$2142 \%$

$24 \%$

$36 \%$

La mayoría de los enfermeros es del sexo femenino, no contrariando el contexto de la enfermería.

Buena parte se refiere casada, predominantemente con más de 36 años de edad. En cuanto al tiempo de formación predomina de 1 a 5 años y 16 a 20, siendo que del total de enfermeros, $52 \%$ se formaron en institución pública y $48 \%$ en privada. La mayoría de los enfermeros es especialista, siendo Máster la mayor titulación referida.

El área de salud se ha desarrollado mucho en los últimos años con la investigación y la tecnología. Así, la cualificación profesional posibilita al profesional de salud mantenerse en constante competitividad en el mercado de trabajo, además de configurarse en busca del 
saber científico que le garantice seguridad en el servicio asistencial, siendo primordial para la emancipación del conocimiento técnico, descubriéndoles un nuevo aprender ${ }^{(18)}$.

En relación al tiempo de servicio, la mayor parte trabaja en la institución de 11 a 15 años, seguida de 1 a 5 años. La mayoría de los entrevistados posee más de un contrato de trabajo, siendo que $80 \%$ posee solo contrato público y $20 \%$ posee contrato público y privado.

El turno de trabajo más citado fue el matutino y vespertino. Sin embargo, por el hecho de que la mayoría trabaja en más de una institución de salud, muchos citaron dos turnos de trabajo y algunos dijeron trabajar en los tres turnos.

El trabajo con carácter de dedicación exclusiva es un factor positivo para el profesional, ya que posibilita la mejora profesional y formación de vínculo tanto organizacional como de usuario. Estudiosos afirman que la precarización de los salarios obliga a los profesionales a tener más de un contrato de trabajo, resultando en carga mensual larga, exhaustiva y desgastante $^{(19)}$, lo que motiva a los profesionales a buscar nuevos conocimientos. Así, hay la necesidad de estimular el carácter dedicación exclusiva, a fin de evitar sobrecarga de trabajo, déficit de atención, fatiga, falta de tiempo para dedicarse a la planificación de la asistencia, así como para mantenerse cualificado.

\section{Eventos adversos: percepción de los enfermeros}

Cuando son preguntados respecto a lo que saben sobre eventos adversos, se observa que la mayoría posee conocimiento: "Lo que puede ocurrir, algo esperado si no se tomaron las medidas necesarias de prevención" (E27); "Lo que no es esperado dentro de la rutina del servicio" (E28); "Son complicaciones derivadas de la realización de cualquier procedimiento realizado en el paciente" (E29); "Son aquellos eventos que aunque esperados, deben ser evitados. Demuestran, muchas veces, incapacidad por parte de quien cuida" (E32); "Son eventos dañinos para el cliente o equipo de trabajo que pueden ser evitados con la adopción de medidas de seguridad" (E6); "Son complicaciones no intencionales provocadas por el funcionario cuando presta cuidado al paciente" (E41); "Son procedimientos erróneos o inadecuados que pueden comprometer la recuperación del paciente, yo considero que son las iatrogenias" (E19).

Sin embargo, otros mostraron que el conocimiento acerca de los eventos adversos es superficial, limitado e inadecuado: "Administración errónea de medicamentos" (E26); "Cualificación" (E42); "Depende del momento" (E4); "Algo que evite daño posterior" (E36).

Y, también, nueve enfermeros no respondieron a la cuestión.

Entender el concepto de evento adverso es el primer paso para saber que el error existe para su reducción.

De acuerdo con la Agencia Nacional de Vigilancia Sanitaria ${ }^{(20)}$, evento adverso es cualquier perjuicio a la salud ocasionado a un usuario o paciente.

En el estudio de Freitas, Oguisso, Merighi( ${ }^{(21)}$ eventos adversos son referidos como ocurrencias éticas, que pueden acarrear alguna forma de perjuicio o daño a los clientes o a los propios profesionales involucrados, sea debido a la falta de atención, de habilidad, de conocimiento, de celo, pudiendo también ser causados por omisión, o sea, cuando el profesional deja de actuar o hacer algo que debería hacer y con eso acarrea riesgo o perjuicio a otro 
Para adecuar el cuidado de enfermería a los paradigmas del siglo XXI es necesario desarrollar, promover y sustentar la retención de profesionales creativos y comprometidos, con habilidad, competencia y responsabilidad profesionales ${ }^{(9)}$.

Los enfermeros precisan conocer para poder prevenir, buscar calidad y fundamentar su acción. Se evidencia la necesidad de capacitar a los enfermeros sobre el concepto y medidas de prevención y control de los EAs, a fin de garantizar una asistencia libre de riesgos y cn calidad.

Gráfico 1 - Eventos adversos ocurridos en un hospital universitario, según la óptica de los enfermeros. Goiânia, Goiás, Brasil, 2010.

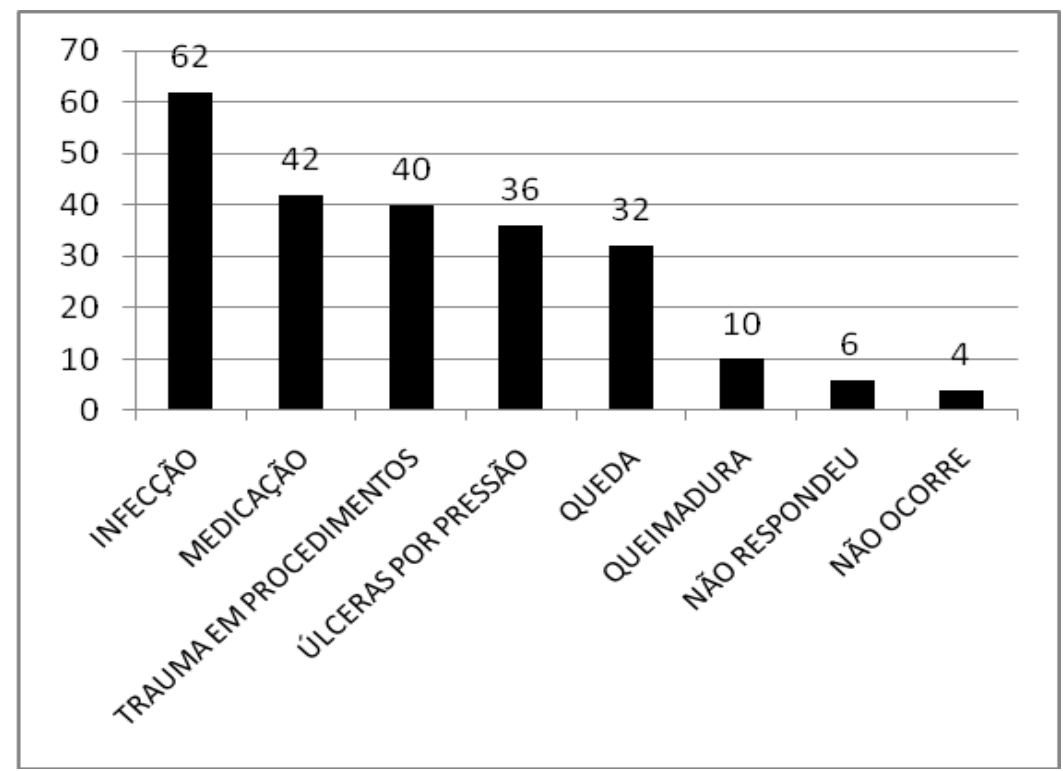

El EA más frecuente en la institución en estudio, según la percepción de los enfermeros, es la infección hospitalaria.

La infección hospitalaria representa un problema de salud pública, además de ser uno de los principales agravantes de la calidad asistencial en salud ${ }^{(22)}$. Este evento adverso prolonga el período de ingreso y es responsable del alto costo del tratamiento para la institución ${ }^{(23)}$.

La infección hospitalaria puede ser de origen endógeno, cuando está causada por microorganismos pertenecientes a la propia flora del paciente o de origen exógeno, cuando es transmitida por las manos de los profesionales de salud $u$ otras personas que entren en contacto con el paciente ${ }^{(24)}$.

De esta forma, cabe a las instituciones de salud elaborar y establecer patrones sistematizados para el manejo del paciente y de materiales, además de establecer estrategias educativas y formación en salud, a fin de reducir la ocurrencia de la infección hospitalaria y evitar los fallos en los procedimientos técnicos.

El segundo evento más relatado fue el error de medicación. Error de medicación es definido como cualquier evento evitable que, de hecho o potencialmente, puede llevar al uso inadecuado de medicamentos ${ }^{(12)}$. 
Según los enfermeros, los errores de medicación más frecuentes son incumplimiento del horario $(17 ; 34 \%)$, error en la dosis $(12 ; 24 \%)$, administración a paciente erróneo $(11 ; 22 \%)$ y medicación no autorizada (6; 12\%).

El resultado encontrado es semejante a otros estudios, que señalan ocurrencias iatrogénicas con medicación, siendo los más frecuentes el incumplimiento del horario, error en la dosificación, medicamento equivocado, vía equivocada, cambio de pacientes, infusión de soluciones incompatibles y aplicación de medicamentos sin autorización ${ }^{(25-26)}$.

Se verifica que el error de medicación está relacionado con los otros errores, destacando el desafío de los profesionales de salud e institución en cuanto a los mecanismos de prevención y abordaje sistémico del error, investigando cada evento para la identificación de las posibles y reales causas.

De esta forma, es necesario un cambio de paradigma, pues no basta que un medicamento tenga garantizada su calidad, sino que su proceso de utilización también debe ser seguro, pudiendo el enfoque sistémico de los errores de medicación revelar fallos del proceso, siendo posible implementar mejoras, disminuyendo así la ocurrencia de esos eventos ${ }^{(12)}$.

Otros eventos destacables fueron los relacionados con los traumas resultantes de procedimentos refiriendo como ejemplos hematomas, dermatitis, accidente con producto químico, escape de aguja durante la punción y, principalmente, los accidentes con perfurocortantes.

Tales accidentes ocurren, en su mayoría, con el equipo de enfermería al manipular objetos perfurocortantes en la realización o auxilio en procedimientos. Algunas causas atribuídas a los accidentes con perfurocortantes son: la disposición de estos materiales en lugares inadecuados 0 en recipientes muy llenos, transporte 0 manipulación de agujas desprotegidas $^{(27)}$.

Las úlceras de presión también fueron citadas por gran parte de los enfermeros. Las escaras o úlceras de presión surgen por la presión sobre prominencias óseas como resultado de la falta rutinaria de cambio de decúbito. Las úlceras de presión han sido consideradas indicadores de calidad de la asistencia de enfermería en las instituciones de salud y su ocurrencia considerada iatrogenia es consecuencia de lo fallos en el proceso de cuidar, dado que existen muchas medidas preventivas para este problema ${ }^{(25,28)}$.

Como el equipo de enfermería presta un cuidado ininterrumpido al paciente, a él se achaca la mayor responsabilidad sobre los cuidados en la prevención de las úlceras de presión. No obstante, para esa prevención es necesario dispensar mayor tiempo al acompañamiento y asistencia de pacientes encamados, y aumento del personal de enfermería.

Las caídas también fueron citadas como evento adverso frecuente y se justifican por el hecho de que el paciente está sin la vigilancia necesaria lo que, sumado a una condición física no adecuada, provoca que muchas veces se levante sin ayuda resultando en caída.

Para evitar que sucedan las caídas pueden tomarse algunas medidas como la elevación de los protectores en las camas y la aplicación de técnicas de restricción de movimientos. La contención en la cama, aunque es vista como una técnica inhumana, cuando realizada correctamente y de forma adecuada no acarrea perjuicios al paciente, configurándose como un excelente medio de prevención de caídas y promoción de la seguridad del paciente ${ }^{(25,26,}$ 29). 
El evento adverso quemadura, a pesar de poco citado, fue referido y su medición debe ser cuidadosa.

Este tipo de evento puede ocurrer más frecuentemente durante la aplicación de compresas calientes que por falta de atención profesional, a la temperatura y al tiempo de exposición del dispositivo en la piel del paciente, lleva a la quemadura. En el caso de pacientes que están obligados a permanecer en cama e inmovilizados, niños y personas con sensibilidad alterada, la atención a este procedimiento debe ser redoblada. Este tipo de evento genera consecuencias para el paciente, siendo que el área lesionada muchas veces puede dejar secuelas. Cada vez que un paciente sufre quemadura, la causa debe ser investigada ${ }^{(25,26)}$.

De esta manera, la ocurrencia de cualquier evento adverso genera daño no sólo a los pacientes, sino también a las instituciones hospitalarias, aumentando el tiempo de ingreso, intervenciones diagnósticas/terapéuticas, exámenes y procedimientos extras, compromiso de la calidad de la atención y de la imagen institucional, desconfianza y aumento de los costos hospitalarios ${ }^{(30)}$ siendo necesaria la adopción de medidas preventivas y cualificación profesional para la comprensión del error en su enfoque sistémico.

A partir de los relatos, se verifica que los enfermeros identifican los eventos adversos y al describir su ocurrencia en la institución, evidencian el desarrollo y compromiso con la asistencia al paciente.

El conocimiento sobre los riesgos para los eventos adversos fue explicitado por la mayor parte de los enfermeros cuando describieron las medidas preventivas adootadas por la institución y equipo de trabajo para posibles correcciones y eliminación de su ocurrencia:

"Uso de EPI, utilización correcta de los equipamientos" (E2); "Comprobación de la posición quirúrgica y de instalación de la placa de bisturí, planes sistemáticos de anotación de informaciones en la recepción/sala operatoria y SRPA" (E6); "Prevención de úlceras de decúbito, prevención de infección, atención a la parada cardiorrespiratoria" (E12); "Administración correcta de medicamentos, cuidados con quimioterápicos y hemoderivados" (E13); "Medidas de control de la infección" (E38); "Técnica aséptica y específica de realización de curativo CDL" (E25); "Lavado de las manos" (E37).

Y, también, se observa la adopción de medidas de acompañamiento, desarrollo y evaluación del servicio para la mejora de la práctica en salud: "Orientación para el personal de limpieza" (E45); "Curso para operadores de autoclaves/montaje de cargas" (E5); "Orientación individual" (E26); "Conferencias explicativas sobre precaución de contacto y control de infección" (E28); "Formación sobre punción venosa" (E29); "Tenemos reuniones mensuales, reuniones diarias [...], oficinas, resolviendo problemas surgidos en el día a día” (E40).

Sin embargo, 23(46\%) enfermeros respondieron que no hay medidas preventivas para la ocurrencia de eventos adversos en la institución o dejaron en blanco la respuesta, evidenciando la necesidad de desarrollar medidas educativas a fin de cualificar a los profesionales de salud y hacerles ver la importancia de actitudes desde simples a complejas para la prevención de los eventos adversos.

La seguirdad de los pacientes en el transcurso de la asistencia de salud es un compromiso ético asumido por los profesionales de enfermería desde su formación y que encuentra en los modelos gerenciales modernos instrumentos para su afirmación. Se manifiesta en quehaceres y actitudes comunes del cotidiano, a través de una simple higiene de manos, de 
orientaciones proporcionadas al paciente y familiares, de la adecuada iluminación y ventilación del ambiente físico, entre otras muchas que, a veces, incluso son percibidas como medidas proactivas de prevención de riesgos ${ }^{(25,31)}$.

Por lo tanto, los resultados señalan la necesidad de desarrollar una cultura de seguridad en el ambiente hospitalario para que el evento adverso sea entendido como parte integrante del sistema, para sistematizar la toma de decisiones frente a la ocurrencia de un evento, así como para asegurar la calidad del servicio de salud.

\section{CONCLUSIÓN}

En el estudio se constató que la mayoría de los enfermeros es de sexo femenino, casada, con edad predominantemente superior a 36 años, con tiempo de servicio en la institución entre 11 y 15 años, siendo la cualificación de Máster la mayor titulación referida.

Se mostró el conocimiento de los enfermeros acerca de los eventos adversos, sin embargo algunos todavía tienen un conocimiento superficial, limitado e inadecuado, lo que dificulta la toma de decisiones y evidencia la necesidad de planificar acciones educativas para la capacitación de estos profesionales respecto al concepto y medidas de prevención y control.

Los eventos adversos más citados fueron infección hospitalaria, seguido de error de medicación, trauma durante procedimientos, úlcera de presión y caída. Algunos no respondieron y otros afirmaron la no ocurrencia de esos eventos en el servicio, deduciendo el alejamiento del enfermero con la práctica gerencial de la asistencia en salud.

A pesar de las dificultades en conceptuar el evento adverso, la mayor parte de los enfermeros reconocieron sus riesgos y señalaron la adopción de medidas de acompañamiento y evaluación del servicio para la mejora de la práctica en salud.

Se espera que el estudio del conocimiento de estos profesionales sobre la ocurrencia de los eventos adversos dentro de la realidad en que están insertos, así como las medidas de seguridad practicadas ayude a las acciones desarrolladas por la enfermería. Entendemos que este estudio moverá a la busca constante de la actualización del conocimiento sobre eventos adversos, a conducir mejor las conductas profesionales para su administración, atendiendo a la mejora de la calidad y de la seguridad de la asistencia prestada.

\section{REFERENCIAS BIBLIOGRÁFICAS}

1. World Health Organizacion - WHO. Word Alliance for Patientt Safety. Forward program 2006-2007 [citado em 23 mai 2010] Disponível em: http://www.who.int/patientysafety.

2. Mills DH, Bolschwing GE von. Clinical risk management. Experiences from the United States. Qual Health Care. 1995; 4(2): 90-6.

3. Wilson R, Runciman WB, Gibberd RW, Harrison BT, Newby L, Hamilton JD. The quality in Australian health care study. Med J Aust. 1995; 163: 458-71.

4. Mendes W, Martins M, Rosenfeld S, Travassos $P$. The assessment of adverse events in hospitals in Brazil. Internatiional Journal for Quality in Health Care. 2009; 21(4): 279-84.

5. Bezerra ALQ, Silva AEBC, Branquinho NCSS, Paranaguá TTB. Análise de queixas técnicas e eventos adversos notificados em um hospital sentinela. Rev. enferm. UERJ, 2009;17(4):467-72.

6. Kohn LT, Corrigan JM, Donaldson MS, editors. To err is human: building a safer health system. Washington, DC: National Academy Press; 2000. 
7. Vincent C, Neale G, Woloshynowych M. Adverse events in British hospitals: preliminary retrospective record review. BMJ. 2001; 322:517-9.

8. World Health Organization. Patient Safety: Rapid Assessment Methods for Estimating Hazards. Genebra; 2003.

9. Pedreira MLG. Enfermagem para segurança do paciente. In: Enfermagem dia a dia: segurança do paciente / organizadores Mavilde da Luz Gonçalves Pedreira, Maria de Jesus Castro Sousa Harada. - São Caetano do Sul (SP): Yendis editora, 2009.

10. Belela ASC. Erros de medicação notificados em uma unidade de cuidados intensivos pediátricos para atendimento de pacientes oncológicos. [Dissertação]. São Paulo: Universidade Federal de São Paulo; 2008.

11. Barbosa LR, Melo MRAC. Relações entre qualidade da assistência de enfermagem: revisão integrativa da literatura. Rev Bras Enferm. 2008; 61(2):366-70.

12. Rosa MB, Perini E. Erros de Medicação: Quem Foi? Rev Assoc Med Bras. 2003; 49(3): 335-41.

13. Pedreira MLG, Harada MCS. Aprendendo aom os erros. In: Harada MJS, Pedreira MLG, editors. O erro humano e a segurança do paciente. São Paulo: Atheneu; 2006. p.175-84.

14. Kusahara DM, Chanes DC. Informes de erros e eventos adversos. In: Enfermagem dia a dia: segurança do paciente / organizadores Mavilde da Luz Gonçalves Pedreira, Maria de Jesus Castro Sousa Harada. - São Caetano do Sul (SP): Yendis editora, 2009.

15. Brasil. Agência Nacional de Vigilância Sanitária. Cartilhas de notificações em tecnovigilância. Ministério da Saúde. Brasília (DF): ANVISA; 2003.

16. Nascimento CCP, Toffoletto MC, Gonçalves LA, Freitas WG, Padilha KG. Indicators of healthcare results: analysis of adverse events during hospital stays. Rev Latino-am Enferm. 2008; 16(4):746-51.

17. Brasil. Conselho Nacional de Saúde. Resolução no 196, de 10 de outubro de 1996.

18. Oliveira NA, Thofehrn MB, Cecagno D, Siqueira HCH, Porto AR. Especialização em projetos assistenciais de enfermagem: contribuições na prática profissional dos egressos. Texto Contexto Enferm. 2009; 18(4):697-704.

19. Nóbrega MFB, Matos MG, Silva LMS, Jorge MSB. Perfil gerencial de enfermeiros que atuam em um hospital público federal de ensino. Rev Enferm UERJ. 2008; 16(3):333-38.

20. Brasil. Agência Nacional de Vigilância Sanitária - ANVISA. Rede Nacional para Investigação de Surtos e Eventos Adversos em Serviços de Saúde. [citado em 15 jun 2011] Disponível em: http://www.anvisa.gov.br/servicosaude/controle/reniss/conceitos.htm 2008.

21. Freitas GF, Oguisso T, Merighi MAB. Ocorrências éticas de enfermagem: cotidiano de enfermeiros gerentes e membros da comissão de ética de enfermagem. Rev. Latino-am Enferm. 2006; 14(4):497-502.

22. Silva RF. A infecção hospitalar no contexto das políticas relativas à saúde em Santa Catarina. Rev. Latino-Am. Enferm. 2003; 11(1):108-14.

23. Brasil - Portaria ํㅡ 2616/MS/GM, de 12 de maio de 1998. Diário Oficial [da] República Federativa do Brasil, Brasília, DF, 13 mai 1998. [citado em: 23 maio 2001]. Disponível em: http://www.anvisa.gov.br/ legis/portarias/2616-98.htm.

24. Carmagnani MIS. Segurança e Controle de Infecção. Rio de Janeiro (RJ): Reichmann e Affonso Editous; 2002.

25. Carneiro

26. Souza

27. Brevidelli MM, Cianciarullo TI. Análise dos acidentes com agulhas em um hospital universitário: situações de ocorrência e tendências. Rev Latino-am Enferm. 2002; 10(6):7806.

28. Carvalho LS, Ferreira SC, Silva CA, Santos ACPO, Regebe CMC. Concepções dos acadêmicos de enfermagem sobre prevenção e tratamento de úlceras de pressão. RBSP. 2007; 31(1):77-89. 
29. Decesaro MN, Padilha KG. Queda: Comportamentos negativos de enfermagem e consequências para o paciente durante o período de internação em UTI. Arq. Ciênc. Saúde Unipar. 2001; 5(2):115-25.

30. Padilha KG, Kitahara PH, Gonçalves CCS, Sanches ALC. Ocorrências iatrogênicas com medicação em unidade de terapia intensiva: condutas adotadas e sentimentos expressos pelos enfermeiros. Rev Escola Enferm. 2002; 36(1):50-7.

31. Padilha KG. Ocorrência iatrogênicas em unidades de terapia intensiva: análise dos fatores relacionados. Rev Paul Enf. 2006; 25(1):18-23. 